\title{
Optimization of Design Parameters of Spiral Spring for Active Headrest Deployment
}

\author{
Yunsik Yang ${ }^{1}$, Euy Sik Jeon ${ }^{2, a}$ and Dae Ho Park ${ }^{1}$ \\ ${ }^{1}$ Department of Mechanical Engineering, Graduate School, Kongju National University, Korea \\ ${ }^{2}$ Department of Mechanical Engineering, (Industrial Technology Research Institute) Kongju National University, \\ Korea.
}

\begin{abstract}
Several studies have been conducted to prevent neck injury in rear-end collision. The headrest of the seat which suppresses the relative motion of the head and the torso can suppress the extension of the head, thereby alleviating the injury. The active headrest has a mechanism that supports the head by deploying the headrest at the rear-end collision. The spring remains compressed or twisted until a collision signal is generated and the headrest is deployed after the collision signal. Depending on the shape and deployment structure of the spring, a spring design with a high resilience that is acceptable to the headrest is required. In this paper, design parameter of spiral spring suitable for the structure of the developed headrest is selected, prototypes are fabricated, and development parameters such as development time and development distance are checked and optimal design parameters of the spiral spring are derived. The feasibility of the headrest with the designed spiral spring was verified by the finite element analysis.
\end{abstract}

\section{Introduction}

In addition to the comfort of passengers, it is essential to ensure safety in order to minimize injury during accidents. It is reported that rear-end collision accident accounts for about $30 \%$ of all traffic accidents and more than $50 \%$ of drivers are suffered neck injury.[1] Several studies have been conducted to prevent neck injuries, and structural studies are under way to control the relative motion of head and body.[2-3] Especially, the headrest of seats is an effective preventive tool to prevent the movement of the head.[4] The deployed headrest has a mechanism that supports the head when the headrest is deployed at the real end collision.[5] Although the driving power source is varied, the structure utilizing the elastic force of the spring is popularly used.[6] The spring remains compressed or twisted until the collision signal is generated, and the headrest is deployed at the same time the deployment signal is generated. The development speed is proportional to the magnitude of the spring force, but it is necessary to design the spring force according to the shape and development structure of the spring to the allowable range of the headrest structure.

In this paper, the design parameters of the spring suitable for the development structure of the developed headrest were selected, and prototypes were fabricated, and the development characteristics such as the development time and the development distance were checked, and the optimum design

\footnotetext{
${ }^{\mathrm{a}}$ Corresponding author : osjun@kongju.ac.kr
} 
factors of the spring were derived. The finite element analysis of the headrest using the designed spring was performed and the structural validity of the headrest was verified by checking the stress distribution.

\section{Mechanism of rear-end collision and neck injury}

A person's neck is an important organ that connects the torso and head. It is a passage through the nerves, and relative movement is possible by the joints and muscles. Sudden shock or vibration can cause pain and damage to muscles and nerves.

The head is anatomically capable of movement such as Flection, Extension, Rotation, and most of the neck injury caused by the Extension of the head in the rear-end collision. As the extension accelerates suddenly in the rearward collision of the vehicle, the upper body of the occupant moves with the seat at the same speed as the vehicle, but the head is not supported firmly with the upper body and moves at a speed 0.5 to 0.6 times slower than the upper body. Therefore, as shown in Figure 1, the upper body and the head are subjected to different movements, resulting in injury.[7-8]

Whiplash was first introduced by Crow in 1982 and generally presents with multiple, complex symptoms such as spinal pain, headache, and speech disturbance due to muscle and ligament strain as a result of deceleration or acceleration in a short time within 50ms or noncontact.[9-10]

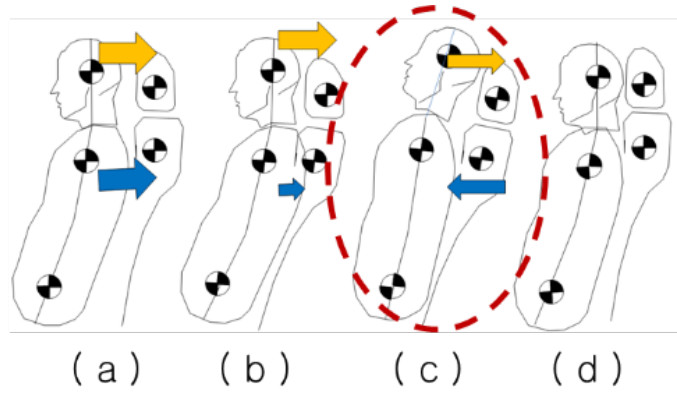

Figure 1. The four phases of head motion as seen from the vehicle headrest

\section{Design of the Headrest structure}

\subsection{Mechanism of headrest}

Figure 2 shows a schematic view of the deployment headrest. Upon receiving the collision signal, the actuator unlocks the spiral spring. The link then rotates and the headrest is deployed. After releasing by the trigger signal, the rotational force of the spring becomes the angular velocity of the end point through the link, which is the deployment speed of the headrest.
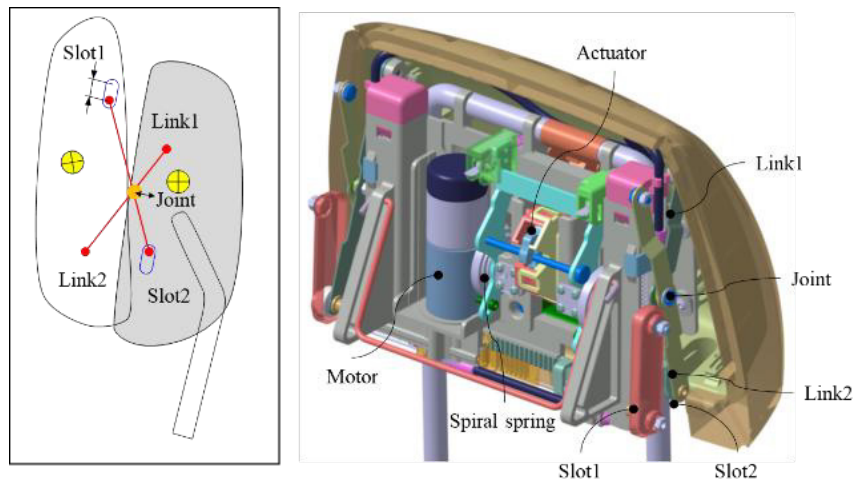
Figure 2. Schematic diagram of the deployment headrest

Figure 3 Is the motion trajectory of the link to select the deployment distance of the deployment headrest.

It expands forward and upward, and the deployment distance is determined by three links. The stroke is moved upward by the rotational force of the spiral spring in a straight line.
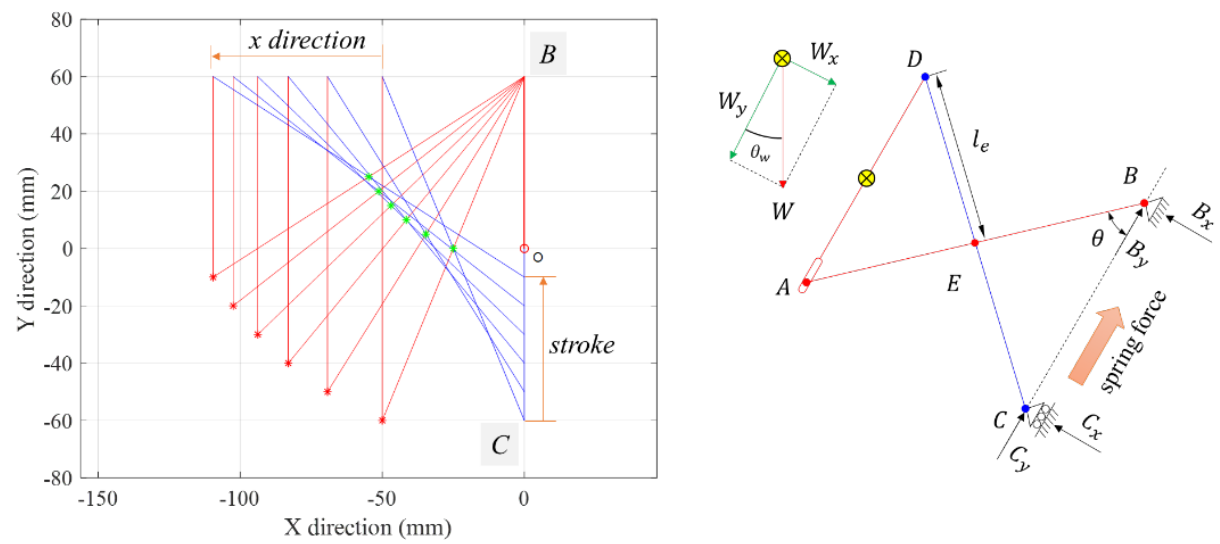

Figure 3. Trajectory analysis and free body diagram for deployment headrest

\subsection{Headrest design parameter}

The designed unfolded headrest is developed by the movement of the crossed links and is influenced by the weight acting on the front headrest according to the tilt angle of the vehicle under free load conditions.

Figure 4 shows the free body diagram of the unfolded headrest. $\mathrm{W}$ is the weight acting on the front headrest, $\theta$ is the expansion angle, and $l_{e}$ is the distance of the link from the cross point.

The point $B$ is fixed and the headrest moves forward when the point $C$ approaches the point $B$ by slid motion due to the elastic force of the spring. The minimum elastic force required for the headrest to deploy is expressed as:

$$
\begin{aligned}
& W=\frac{W_{x}}{\sin \theta_{w}}=\frac{W_{y}}{\cos \theta_{w}} \\
& C_{y}=\frac{-W_{x}}{\tan \theta}=\text { spring force } \\
& C_{x}=W_{y} \tan \theta-\frac{W_{x}}{2} \\
& B_{y}=\frac{W_{x}}{\tan \theta}+W_{y}
\end{aligned}
$$

At this time, friction occurring in each joints and slots was ignored. 

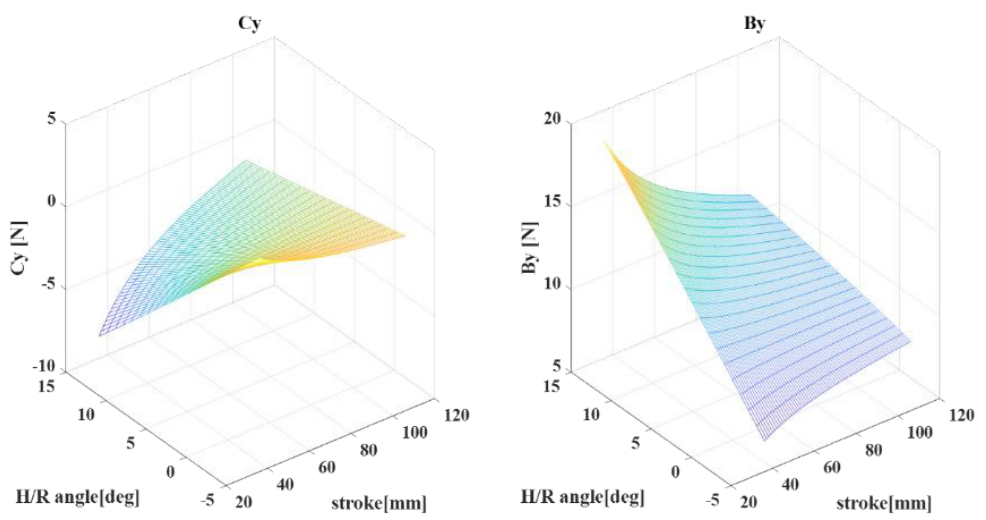

Figure 4. Change of elasticity according to headrest deployment angle and deployment distance

As shown in Figure 4, the change of the minimum required elasticity according to the change of headrest deployment angle and deployment distance was confirmed.

The range of the elastic force was selected by applying the safety factor to the derived elastic force in consideration of the dynamic motion and the friction coefficient.

\section{Theoretical analysis of expansion spring}

\subsection{Theoretical analysis of spiral spring}

Spiral springs are generally used because they can efficiently utilize the characteristics and space using twist. One end is fixed and the other end is twisted to use the spring action by the twist deformation at that time. The elastic energy to be stored per unit volume is large and lightweight, does not take up much space, has a simple shape, and has an advantage that the spring characteristic is in good agreement with the calculated value. Since the linear relationship can be maintained in the relationship between the torsion angle and the torque, the formula considering various conditions should be used.
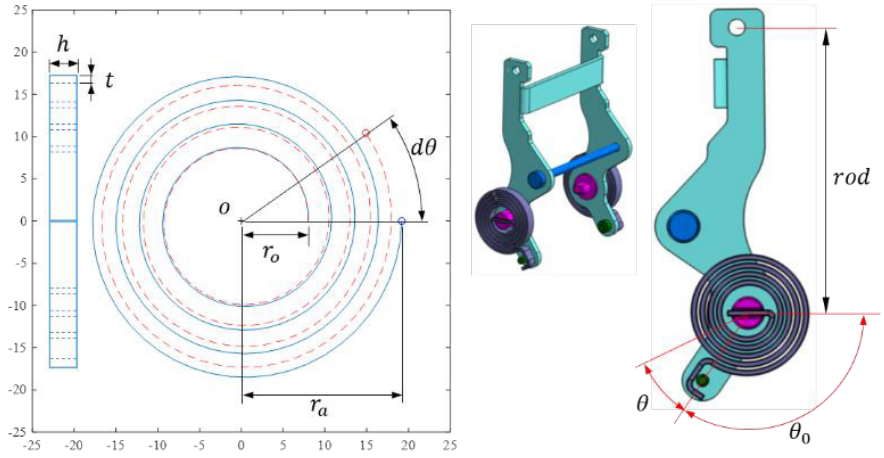

Figure 5. Shape of spiral spring and active mechanism

As shown in Figure 5, $r=m\left(\theta+\dot{\theta}_{0}\right)+r_{0}$ where $r_{0}$ is the inner diameter of the spiral portion of the spiral spring, and $r_{a}$ is the distance from the center $O$ to the outer end. Where, $m=\frac{p}{2 \pi}, \mathrm{P}$ is the pitch, $\theta_{0}$ is the angle(rad) from the position fixed to the inner shaft of the spiral to the $x$-axis measured in the counter clockwise direction, and the length $l$ is expressed as follows.

$$
L=\int_{0}^{L} d s=\int_{0}^{L} \sqrt{(d r)^{2}+(r d \theta)^{2}}=\int_{0}^{\theta_{a}} \sqrt{m^{2}+r^{2}} d \theta
$$


The strain angle $\theta$ can be expressed as follows by the Castilian's Theorem.[11]

$$
U=\int \frac{M^{2} d x}{2 E I}
$$

Where, $M=F r$, and the deformation angle of the end is expressed as follows.

Where, $I=\frac{h t^{3}}{12}$

$$
r \theta=\frac{\partial U}{\partial F}=\int_{0}^{l} \frac{\partial}{\partial F}\left(\frac{F^{2} r^{2} d x}{2 E I}\right)=\int_{0}^{l} \frac{F r^{2}}{E I} d x
$$

$$
\begin{aligned}
& d \theta=\frac{12 M L}{E h t^{3}} \frac{180}{\pi} \\
& k=\frac{M}{d \theta}=\frac{E h t^{3}}{12 L} \frac{\pi}{180}
\end{aligned}
$$

The elastic force of the torsion spring is proportional to the third square of the thickness and is inversely proportional to the length, so it is selected as the main factor in designing and manufacturing the spring.

\subsection{Design parameters of spiral spring affecting moment}

The deployed headrests are typically powered by a spring-loaded structure, because it is simple to structure and allows for high-acceleration movements of large loads with very high loads in a very short time. The elasticity of the spiral spring and the length of the rod determine the deployment speed and deployment distance. It is very important to analyse and optimize the dynamic characteristics of the spring, because the dynamic performance of the spring determines the expansion performance of the headrest.

\section{Analysis of headrest deployment test}

\section{1 headrest deployment test}

Since the spring rotates momentarily in a twisted state, it is necessary to analyze the dynamic behavior of the spring as a test.

In this study, a simple product was made to confirm that the headrest was developed by a spiral spring. As shown in Figure 6, the model of the spiral spring was fabricated using pw-2 material. The inner diameter was fixed, and the test piece was fabricated by changing the moment of inertia, effective winding number and outer diameter

Fix the notch rod of the headrest and give a signal to the actuator to analyse the dynamic behaviour using a high-speed camera when the spring rotates and the link rotates at high speed. As shown in Figure 7, the development of the headrest was taken at $1000 \mathrm{fps}$. The measurements were made under the same conditions and showed errors within $2 \%$.
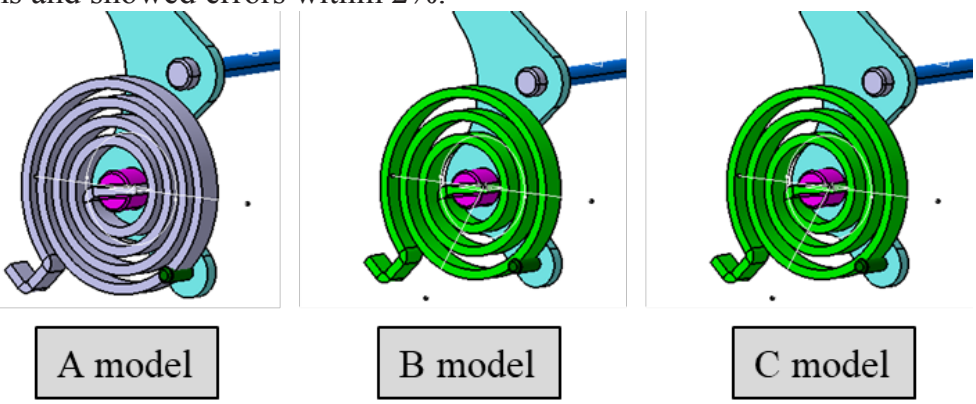

Figure 6. Model for spiral springs 

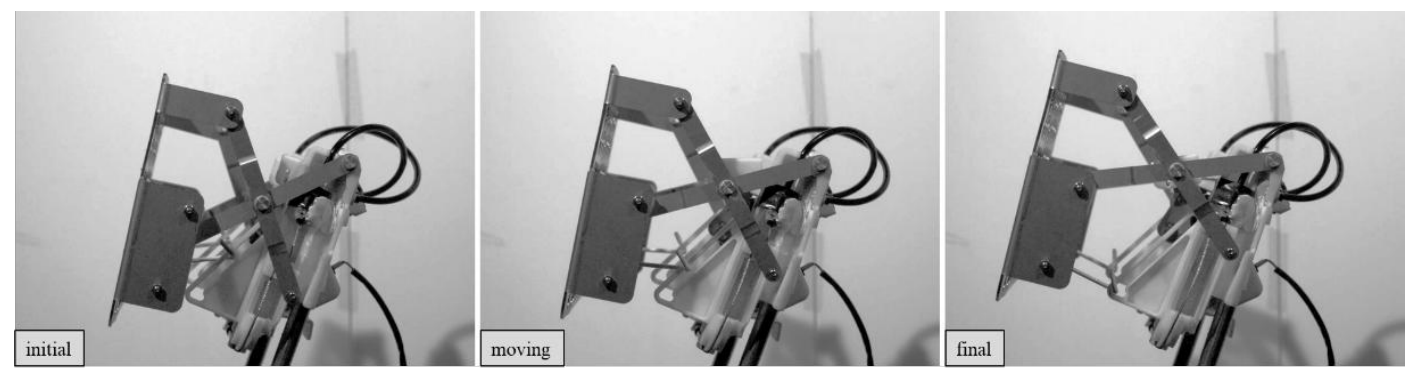

Figure 7. Active headrest motion

\subsection{Analysis of Relation between Deployment Speed and Moment}

When the headrest was deployed, the displacement and the deployment time of the headrest end of the test were confirmed. It is assumed that the moment acting on each part is constant and no external force other than moment acts.

As shown in Table 1, the development time is inversely proportional to the moment as the moment of each specimen increases. Overall, it was confirmed that the shape of the prototype was inversely proportional to the design parameters.

In the theoretical analysis, the stiffness coefficient is found to be proportional to the moment of inertia and inversely proportional to the effective length, number of active coils. The design parameters of the spiral spring were selected as shown in Figure 8, and the range of the development time was estimated according to the change of the moment.

Table 1. Experimental conditions for spiral spring

\begin{tabular}{|c|c|c|c|c|}
\hline Element & Unit & A model & B model & C model \\
\hline Hight $(\mathrm{h})$ & $\mathrm{mm}$ & 5 & 5 & 5 \\
\hline Thickness $(\mathrm{t})$ & $\mathrm{mm}$ & 2.0 & 2.0 & 2.2 \\
\hline Number of active coil & - & 4 & 3 & 3 \\
\hline Moment & $\mathrm{Nm}$ & 43 & 101 & 134 \\
\hline Active time & $\mu s$ & 100 & 40 & 30 \\
\hline
\end{tabular}

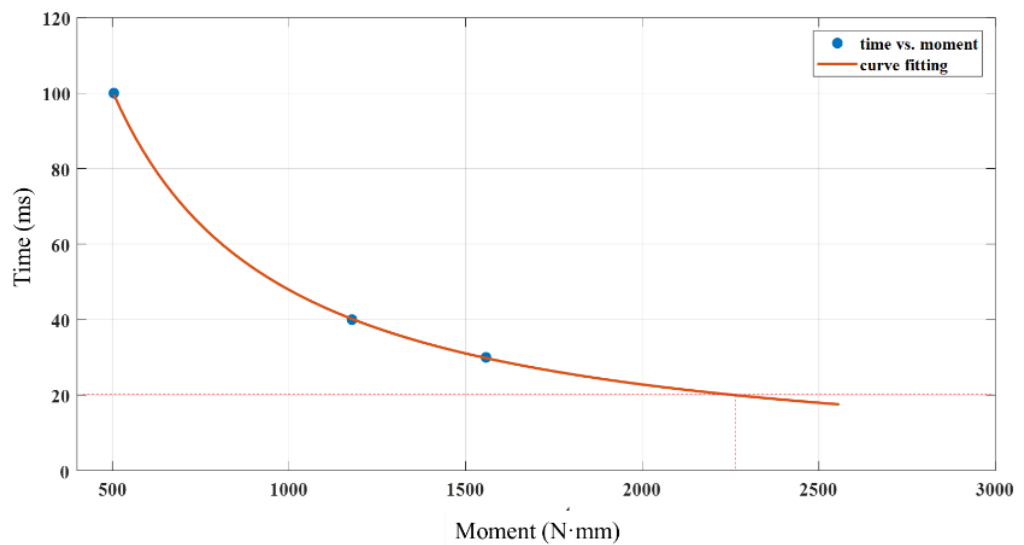

Figure 8. Estimation of deployment time using moments 
In order to obtain the value of the experimental value close to the theoretical value, different tests such as the moment of inertia, length, and number of active coils were performed.

From the experimental results, we can confirm the power approximation function such as Equation 10 using the target development time and spiral spring moment and set the design goal of the spring that satisfies the development time of $20 \mathrm{~ms}$ or less.

$$
y=77654.69 x^{-1.07}
$$

Based on the test results, we will use torsion and torque as data to estimate the design factors affecting each deployment time.

\section{Finite element analysis}

Structural analysis was performed to determine the effect on the headrest body when spiral spring was selected according to the range of design parameters. As Shown in Figure 9, the elastic force and the stresses distribution of the supporting structure are confirmed and the fatigue characteristics at the repeated operation are also confirmed. Finite element analysis showed no effect on the headrest frame.
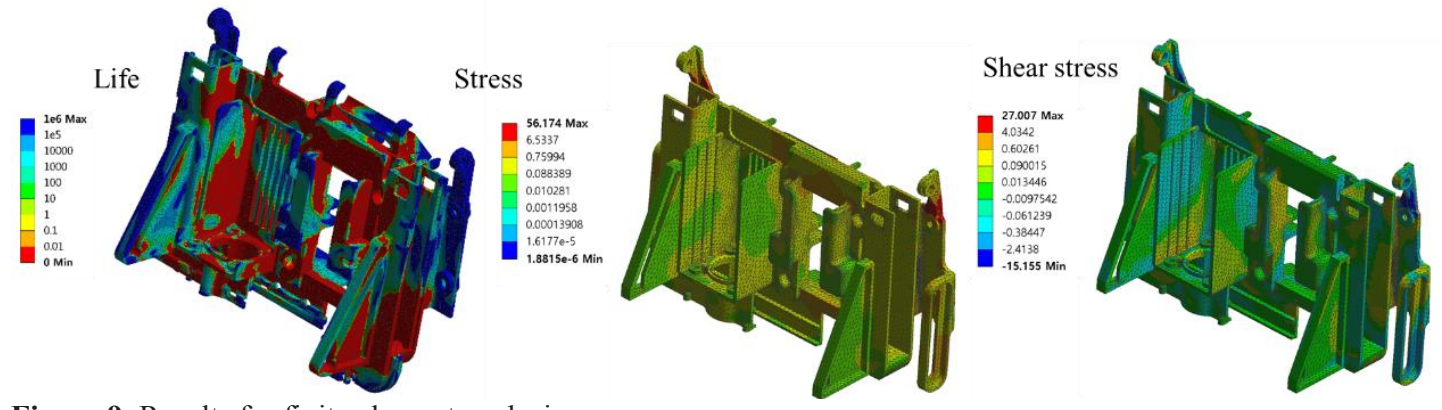

Figure 9. Results for finite element analysis

\section{Conclusions}

We confirmed the headrest deployment characteristics to mitigate neck injuries and confirmed the design parameters. Through the kinematic analysis of the active headrest, the proposed design parameters were confirmed and the development structure was designed based on this.

The design parameters of the spiral spring for the headrest to accurately and quickly support the head of the passenger are proposed. Spiral spring and headrest prototypes were fabricated and a simple test was carried out to confirm the design range of the spring. The designed headrest was subjected to a structural analysis of the corresponding spring to verify the design validity of the headrest.

\section{ACKNOWLEDGEMENTS}

This research was supported by the Ministry of Trade, Industry \& Energy(MOTIE), Korea Institute for Advancement of Technology(KIAT) through the Encouragement Program for The Industries of Economic Cooperation Region(R0004149)

\section{References}

1. P. Ardoino, Whiplash injuries, (1996)

2. W. H. Castro, M. Schilgen, S. Meyer, M. Weber, C. Peuker, K. Wortler, whiplash injuries, 6, 6 (1997) 
3. R. M. Greendyke, Jama, 195, 7 (1966)

4. A. J. Watts, D. Atkinson, C. J. Hennessy, Lawyers \& Judges Publishing Co., Tuscon, USA (1996)

5. A. Nygren, H. Gustafsson, C. Tingvall, (1985)

6. M. Panjabi, J. Wang, N. Delson, Proceedings of the International Research Council on the Biomechanics of Injury Conference, 27 (1999)

7. H. Fischer, H. Malsch, Active Headrest for a Vehicle Seat, (2003)

8. M. Farquhar, M. Humer, K. Grewal, K. Low, K. McQueen, N. V. Yetukuri, (2004)

9. J. Schilling, M. Böhmer, D. Hippel, D. Wrobel, H. Klein, G. Ruess, et al., (2006)

10. K. Boes, M. Schmid, T. Frank, A. Tscherbner, (2011)

11. J. Chen, I. Chen, Int. J. of Solids and Structures, 64 (2015) 